\title{
Toxic Shock Syndrome in Hospitalized Patients with Staphylococcus aureus Infection
}

As a consequence of a broader epidemiologic study of toxic shock syndrome (TSS) (1), it became clear that many hospitalized cases of TSS could be related to diseases, both major and minor, involving Staphylococcus aureus infection. We, therefore, investigated the incidence of TSS in hospitalized patients with suspected or proven $S$. aureus infection.

To identify charts for review, we developed a set of all International Classification of Diseases, Adapted (ICDA) (2) codes (available upon request) that might be used to code for $S$. aureus-associated disease. At five Denver area hospitals (four general community hospitals and the fifth a children's hospital), we reviewed all available medical records having those codes for all even years, 1970 to 1980 inclusive, to identify definite or possible cases of $S$. aureus disease and cases of TSS. The latter were identified using our previously reported and validated screening and strict case definitions of TSS (3). $S$. aureus cases were divided into "definite infection" and "possible infection" categories by one of us (AMW). A case was considered "definite" if the $S$. aureus was obtained from a non-permissive, inflamed site (blood, bone, abscess cavity, etc) and "possible" if the organism was cultured from a mucosal or skin surface adjacent to an inflamed site or directly from a wound lacking in gross signs of purulence.

3,353 of $3,625(92.5 \%)$ selected medical records were actually available for review. Five hundred and sixty-three $S$. aureus-associated cases were identified (see Table 1). Eight of these conformed to the TSS screening definition alone (four females and four males), and eight satisfied the terms of the strict defintion of TSS (seven females and one male). The combined rate of TSS per 100 hospitalized $S$. aureus cases ranged from 0 to 5.0 at the various hospitals with a mean of 2.4. At least one case of TSS occurred in every year investigated. Six of the 16 cases were felt to be "definite infection" as opposed to ten "possible", including all cases with positive vaginal cultures (by definition). There were three females in the "definite infection" group (two with $S$. aureus bacteremia and one with a buttock abscess) who were greater than ten years of age, and two of them were noted to be menstruating during their hospitalization while the menstrual status of the third, a 15-year-old with bacteremia, was not noted in the medical record.

Underlying the study was the assumption that a proportion of hospitalized patients with definite or possible $S$. aureus disease should have manifested the signs and symptoms of TSS if the capacity to elaborate the causative toxin(s) was
Table 1: Incidence of toxic shock syndrome in patients thought to have focal Staphylococcus aureus infections.

\begin{tabular}{|c|c|c|c|c|}
\hline & clinical ISS & per no. Staphy & lococcus our & sepisodes \\
\hline & & 1. : Possibl & e infection & \\
\hline & Definite & Unsuspected & Suspected & s. \\
\hline Year & Infection & TSS & TSS* & All cases: \\
\hline 1970 & $1 / 62(0)^{* *}$ & $1 / 26(0)$ & & $2 / 88(0)$ \\
\hline 1972 & $2 / 73(1)$ & $1 / 26(0)$ & & $3 / 99$ (1) \\
\hline 1974 & $2 / 69(1)$ & $0 / 16(0)$ & & $2 / 85(1)$ \\
\hline 1976 & $0 / 85$ & $2 / 26(0)$ & & $2 / 111(0)$ \\
\hline 1978 & $1 / 72(0)$ & $0 / 19$ & & $1 / 91(0)$ \\
\hline 1980 & $0 / 65$ & $1 / 19(1)$ & $5 / 5(5)$ & $6 / 89(6)$ \\
\hline Totals & $6 / 426(1.4 \%)$ & $5 / 132(3.8 \%)$ & $5 / 5(100 \%)$ & $16 / 563(2.8 \%)$ \\
\hline
\end{tabular}

* Menstruating women thought to have TSS and subsequently cultured seeking an $S$. aureus focus;

** $(\quad)=$ number of cases by only the strict case definition.

reasonably prevalent among those bacteria. That assumption was supported by our findings - that the incidence of 2.8 cases of TSS per 100 patients with definite or possible $S$. aureus infection proved to be relatively uniform from year to year. This incidence $(2,800 / 100,000$, or $1,400 / 100,000$ if only the strict case definition is used) is several orders of magnitude greater than the maximum incidence we have estimated for the general population $(9.1 / 100,000)$, or even for females of menstrual age $(15.8 / 100,000)(1)$ and suggest that high-risk clinical groups for which TSS is suspected (menstruation plus tampon, shock, febrile mucocutaneous illness [3]) should include hospitalized patients with suspected $S$. aureus infection.

\section{A.M. Wiesenthal, M. Ressman, S.A. Caston, J.K. Todd}

\section{Literature}

1. Todd, J. K., Wiesenthal, A. M., Ressman, M., Caston, S. A., Hopkins, R. S.: Toxic shock syndrome - II. Estimated occurrence in Colorado as influenced by case ascertainment methods. Am. J. Epidemiol, 122 (1985) 847-856.

2. Commission on Professional and Hospital Activities. Hospital Adaptation of International Classification of Diseases, Adapted, Volume I. First edition (November 1968), second edition (September 1973); and Commission on Professional and Hospital Activities. International Classification of Diseases, 9th revision. Clinical Modification (January 1979).

3. Wiesenthal, A. M., Ressman, R., Caston, S. A., Todd, J. K.: Toxic shock syndrome - I. Clinical exclusion syndromes by strict and screening definitions. Am. J. Epidemiol. 122 (1985) 857-867.

Received: 17 September 1985/Accepted: 3 February 1986

A. M. Wiesenthal, M.D., Marilyn Ressman, R.N., Sharon A. Caston, R.N., J. K. Todd, M.D., Department of Infectious Disease, The Children's Hospital, 1056 East 19th, Denver, Colorado 80218, U.S.A.

Correspondence to: J.K. Todd, M.D. 